\title{
Co-culture of adipocytes with macrophages inhibits insulin action and promotes a pro-inflammatory state in adipocytes that is modulated by long-chain $n$-3 PUFA
}

\author{
E. Oliver ${ }^{1,2}$, C. Phillips ${ }^{1}$, S. Toomey ${ }^{1}$ and H. M. Roche ${ }^{1}$ \\ ${ }^{1}$ Nutrigenomics Research Group, UCD Conway Institute, University College Dublin, Republic of Ireland and \\ ${ }^{2}$ Department of Clinical Medicine, Institute of Molecular Medicine, Trinity College Dublin, Republic of Ireland
}

\begin{abstract}
Obesity is the key aetiological factor that predisposes individuals to insulin resistance. Recent studies have shown that obese adipose tissue is characterised by increased infiltration of macrophages ${ }^{(1)}$. These macrophages may release pro-inflammatory stressors, which may alter insulin sensitivity in adipocytes by modifying the insulin-signalling pathway, leading to type 2 diabetes mellitus (T2DM). There is strong evidence to suggest that long-chain (LC) $n$-3 PUFA have anti-inflammatory effects ${ }^{(2)}$. The present study has investigated the molecular interactions between macrophages and adipocytes to ascertain these effects on molecular markers of insulin sensitivity and inflammation in adipocytes and lastly to determine the therapeutic effects of anti-inflammatory EPA and DHA on these markers.

A co-culture of differentiated 3T3-L1 adipocytes and J774.2 macrophages was performed. In the contact system 3T3-L1 cells were cultured with unstimulated and lipopolysaccharide (LPS; $0.1 \mu \mathrm{g} / \mathrm{ml}$ )-stimulated J774.2 cells for $24 \mathrm{~h}$ followed by EPA or DHA (50 $\mu \mathrm{mol} / \mathrm{l}$ ) treatment for a further $24 \mathrm{~h}$. In the trans-well system inserts kept the cell types separate but allowed exchange of soluble molecules. Cultured cells and supernatant fractions were harvested for RT-PCR, Western blot, and ELISA.

The presence of macrophages significantly reduced adipocyte GLUT4 and insulin receptor substrate-1 (IRS-1) mRNA, classical markers of insulin sensitivity. In contrast, inflammatory markers IL-6, TNF $\alpha$ and TLR4 mRNA levels were up regulated by the presence of macrophages. The presence of LPS stimulated macrophages significantly increased NF- $\kappa \mathrm{B}$ and significantly reduced its inhibitor IKB $\alpha$ within the adipocytes. Adipocytes co-cultured with LPS-stimulated macrophages expressed significantly less GLUT4 and IRS-1 protein while JNK1 an inhibitor of IRS-1 function was significantly increased. Cell supernatant TNF $\alpha$, IL- 6 and IL- $1 \beta$ concentrations were increased while adiponectin levels were reduced on exposure to the macrophages. Interestingly, EPA and DHA modified these effects within the adipocytes, reducing expression of pro-inflammatory mediators (TNF $\alpha$, IL-6, IL-1 $\beta$, JNK1, NFKB while increasing IKB $\alpha$ ) and increasing markers of insulin sensitivity (GLUT4 and IRS-1).

These results suggest that obesity is associated with the generation of macrophage-derived soluble factors that promote a proinflammatory insulin-resistant adipocyte function. The work shows that pre-culturing macrophages with EPA and DHA can attenuate this effect. A longer pretreatment of the macrophages with LC $n$-3 PUFA may be necessary to see more significant effects. Further work is continuing to elucidate the molecular interactions between adipocytes, macrophages and dietary fatty acids.
\end{abstract}

E. O. is a recipient of the PhD in Molecular Medicine and the HRB PhD Training Site Programme at Trinity College Dublin. This work was supported by the European Commission, Framework Programme 6 (LIPGENE): contract no. FOOD-CT-2003-505944.

1. Weisberg S, McCann D, Desai M, Rosenbaum M, Leibel R \& Ferrente A (2003) J Clin Invest 112, 1796-1808.

2. Weldon S, Mullen A, Loscher C, Hurley L \& Roche H (2006) J Nutr Biochem 18, 658-666. 